\title{
Loss of T-Cadherin (CDH13, H-Cadherin) Expression in Cutaneous Squamous Cell Carcinoma
}

\author{
Tamotsu Takeuchi, Sheng-Ben Liang, Norihisa Matsuyoshi, Shuxia Zhou, \\ Yoshiki Miyachi, Hiroshi Sonobe, and Yuji Ohtsuki \\ Department of Pathology (TT, HS, YO), Kochi Medical School, Kochi, and Department of Dermatology (NM, SZ, \\ YM), Graduate School of Medicine, Kyoto University, Kyoto, Japan; and Division of Experimental Therapeutics \\ (S-BL), Ontario Cancer Institute, Toronto, Canada
}

\begin{abstract}
SUMMARY: We previously reported that T-cadherin (CDH13, H-cadherin), a unique cadherin molecule, was expressed on the basal cell layer in normal murine and human epidermis. In the present study, T-cadherin expression in archival human skin specimens comprising a spectrum of human squamous cell neoplasia was investigated. T-cadherin expression was observed in both normal epidermal basal cells and adnexal epithelial cells of formalin-fixed and paraffin-embedded tissue sections. Western immunoblotting also revealed that mature T-cadherin protein was expressed in cultured human skin tissue equivalent. Atypical keratinocytes in 27 of 53 specimens of actinic keratosis and 23 of 30 specimens of Bowen's disease expressed T-cadherin. In contrast, T-cadherin was focally expressed in 6 of 56 invasive cutaneous squamous cell carcinomas. To explore the molecular mechanism of down-regulation of T-cadherin expression in invasive squamous cell carcinoma, loss of heterozygosity, genetic alternations, and methylation status in the $5^{\prime}$ region of the T-cadherin gene were investigated. Loss of heterozygosity at intron 1 of the T-cadherin gene was observed in 8 of 28 informative cases of invasive squamous cell carcinoma. Although no structural genomic alternations were found by sequence analysis, aberrant promoter methylation of the T-cadherin gene was found in 12 of 28 invasive squamous cell carcinomas. T-cadherin expression was restored in cultured A431 cells, in which aberrant methylation was found by treatment with the demethylating agent 5'-aza-2-deoxycytidine. These findings suggest that a combination of deletion and aberrant methylation of the T-cadherin gene may play a role in loss of gene expression in a considerable number of invasive cutaneous squamous cell carcinomas. (Lab Invest 2002, 82:1023-1029).
\end{abstract}

$A$ ctinic keratosis and Bowen's disease are characterized by an epidermis that is thickened by atypical keratinocytes proliferating at lower levels and at all levels, respectively, of the epidermal layer. Actinic keratosis has traditionally been regarded as "squamous cell carcinoma, grade 1/2" and is histologically characterized by dysplasia of the lower epidermal layers, first in the basal cell layer and gradually progressing to the overlaying layer.

Bowen's disease is a malignant proliferation of epidermal cells in all layers of the epidermis and usually exhibits prolonged periods of intraepidermal growth. Detachment of tumor cells from the epidermis is believed to be an essential step of invasion or metastasis of these squamous cell neoplasms. It is believed that down-regulation or aberrant expression of cellular adhesion molecules of atypical keratinocytes plays a role in this critical initial invasion (Cerri et al, 1994; Llorens et al, 1998; Pizarro, 2000). Exploration of the molecules that are down-regulated in

\section{DOI: 10.1097/01.LAB.0000025391.35798.F1}

Received February 28, 2002.

This study was supported by grants from the Ministry of Education of Japan and the Medical Research Fund of Kochi Medical School.

Address reprint requests to: Dr. Tamotsu Takeuchi, Department of Pathology, Kochi Medical School, Nankoku, Kochi, Japan 783-8505. E-mail: takeutit@med.kochi-ms.ac.jp invasive squamous cell carcinoma is thus of considerable importance.

Very recently, we reported that $\mathrm{T}$-cadherin, identical to $\mathrm{H}$-cadherin or $\mathrm{CDH} 13$, is present on the basal cell layer of mouse and human skin (Zhou et al, 2002). T-cadherin was located on both cell-cell contacts and the basal side of the epidermal basal cells. E- and P-cadherins, the other cadherin molecules expressed in epidermis, are classical cadherins and have cytoplasmic domains interacting with catenins and the actin intracellular filament network. In contrast, T-cadherin is a nonclassical cadherin that binds to surface membranes without a cytoplasmic domain through glycosyl phosphatidyl inositol-linker (Nagafuchi and Takeichi, 1988; Ranscht and DoursZimmermann, 1991). Recent studies suggest that T-cadherin may be involved in fundamental processes of intracellular signaling as well as cell-cell adhesion (Fredette et al, 1996; Takeuchi et al, 2000, 2001a; for review, see Takeuchi and Ohtsuki, 2001c). Moreover, down-regulation of T-cadherin seems to play a role in inducing malignant phenotypic change and tumorigenicity in breast, lung, ovary, and bladder cancers (Kawakami et al, 1999; Lee, 1996; Maruyama et al, 2001; Sato et al, 1998a; Toyooka et al, 2001).

In the present study, we examined T-cadherin expression in archival human tissues that comprise a spectrum of human squamous cell neoplasia. Interestingly, T-cadherin expression was down-regulated in a 
considerable number of invasive squamous cell carcinomas by the combination of aberrant methylation and gene deletion, as observed for breast and lung cancers.

\section{Results and Discussion}

Recently we demonstrated that the basal cell layer of human and murine skin was stained with affinitypurified rabbit anti-T-cadherin peptide antibody by using cryosections (Zhou et al, 2002). To examine the T-cadherin expression in pathologically stocked skin tissue sections, we first explored the methods for antigen retrieval. After autoclave pretreatment, T-cadherin expression was observed in normal human epidermal basal cells of $10 \%$ buffered formalin-fixed and paraffin-embedded tissue sections as previously found in cryosections (Fig. 1A). Preadsorption of antibody with T-cadherin peptide diminished this staining ( Fig. 1B). The outer root sheath of hair follicles was also stained with anti-T-cadherin antibody (Fig. 1, C and $D$ ). Keratinocytes in the isthmus and infundibulum of hair follicles and sebaceous glands were stained with anti-T-cadherin antibody (Fig. 1E). Basal cells of eccrine glands were also stained with anti-T-cadherin antibody (Fig. 1F). Interestingly, T-cadherin was expressed in the cytoplasm of basal cells of eccrine glands. Staining in eccrine glands was also diminished by preincubating the antibody with T-cadherin peptide (data not shown).

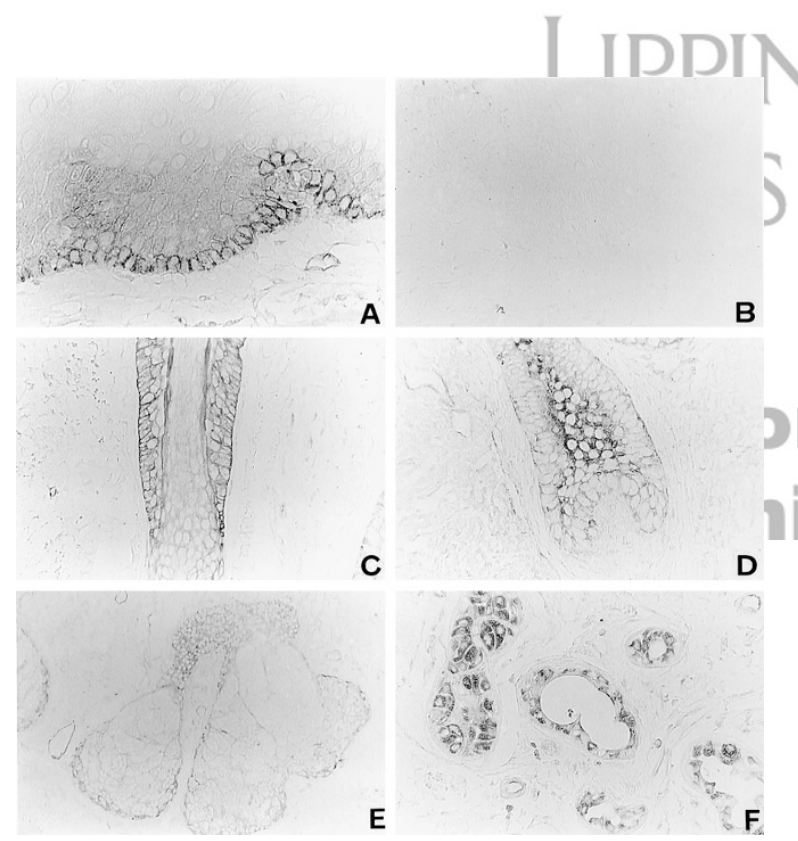

Figure 1.

T-cadherin expression in noncancerous human skin. Formalin-fixed and paraffin-embedded human skin tissue was immunohistochemically stained with affinity-purified rabbit antibody to human T-cadherin peptide. The epidermal basal cells were stained by the antibody $(A)$. Note the staining on both cell-cell contacts and the basal side of the basal cells of the skin. Preadsorption with T-cadherin peptide eliminated all staining of epidermal basal cells (B). Hair follicles, especially their outer root sheaths, were also stained with the antibody ( $C$ and $D)$. Ducts and mature sebocytes of sebaceous glands $(E)$ and basal cells of eccrine glands $(F)$ were also stained with anti-T-cadherin antibody. Original magnifications, $\times 200(A$ to D, F); $\times 100(E)$.
Cytoplasmic expression in eccrine glands suggests that T-cadherin may have effects apart from those on cellular adhesion. Western immunoblotting previously revealed that human keratinocyte DJM-1 cells (Kitajima et al, 1987) expressed 90-kDa T-cadherin protein (Zhou et al, 2002). We used a commercially available antibody in the previous Western immunoblotting. In the present study, specificity of the anti-T-cadherin antibody prepared in our laboratory was confirmed by Western immunoblotting as described below. Human cultured skin tissue equivalents were lysated and subjected to SDS-PAGE followed by immunoblotting. A single major band with an approximate molecular weight of $90 \mathrm{kDa}$ was detected in lysates of human skin tissue equivalents as observed in DJM-1 cells (Fig. 2A). Notably, two major 90- and 100-kDa proteins were detected in human heart lysates (Fig. 2B). Although the functional differences between the 90 - and $100-k D a$ proteins remain unclear, the larger $100-k D a$ $\mathrm{T}$-cadherin protein is believed to be a precursor protein of the 90-Da T-cadherin molecule (Fredette et al, 1996). Therefore, the rabbit antibody used in the present study detected mature T-cadherin protein in organ-cultured human skin tissues. Immunohistochemical staining also revealed expression of T-cadherin in the basal cell layer of cultured skin tissues, as observed in the surgically resected skin specimens (Fig. 2E). Combined with the results of immunohistochemical staining with preadsorbed antibody (Fig. 1B), it is clear that the antibody used in this study specifically reacted with T-cadherin molecule in autoclave-pretreated archival tissue sections.

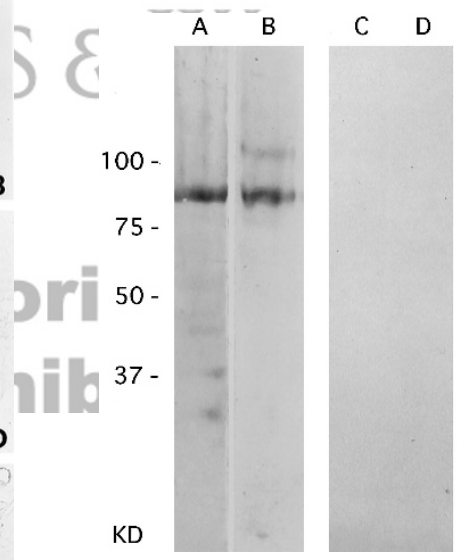

E

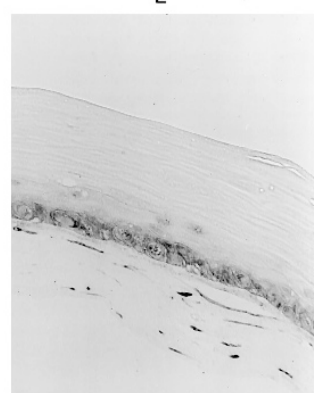

Figure 2.

Western blot analysis of lysates of cultured human skin tissue and immunohistochemical staining of cultured human tissue. Cell lysates of cultured human skin tissue (lanes $A$ and $C$ ) and human heart extracts (lanes $B$ and $D$ ) were subjected to SDS-PAGE and transferred to membrane. Lanes $A$ and $B$ were reacted with affinity-purified rabbit antibody to human $T$-cadherin peptide, whereas lanes $C$ and $D$ were reacted with control rabbit IgG. $A$ band with an approximate molecular weight of $90 \mathrm{kDa}$ was detected in the human cultured skin tissue with anti-T-cadherin antibody (lane A). Two major bands with approximate molecular weights of 90 - and $100-\mathrm{kDa}$ were detected in human heart extracts with anti-T-cadherin antibody (lane $B$ ). No significant band was observed for either human skin lysates or heart extracts with control antibody (lanes $C$ and $D$, respectively). Cultured human skin tissues were also formalin-fixed and paraffin-embedded and immunohistochemically stained with antibodies. Epidermal basal cells were stained with rabbit anti-T-cadherin antibody. Original magnifications, $\times 400$. 
Next, immunohistochemical staining of precancerous and cancerous skin tissue was performed. The results are summarized in Table 1A. Atypical keratinocytes of 27 of 53 specimens of actinic keratosis and 23 of 30 specimens of Bowen's disease were strongly stained with anti-T-cadherin antibody (Fig. 3, A to D). The finding that both the stratum basale (germinativum) of noncancerous skin and proliferating atypical keratinocytes or tumor cells in the epidermis expressed T-cadherin protein suggests that T-cadherin may play an important role in intradermal keratinocyte growth. Further immunohistochemical staining revealed that 6 of 56 invasive squamous cell carcinoma specimens were focally stained with anti-T-cadherin antibody (Fig. 3E). However, invasive squamous cell carcinoma cells of 50 of 56 specimens did not express T-cadherin (Fig. 3F). Interestingly, actinic keratosis components, from which invasive squamous cell carcinoma arose, did not express T-cadherin molecule. Silencing of the T-cadherin molecule could be an event that precedes cancer invasion.

Recent studies have revealed that T-cadherin may function as a tumor suppressor factor in various cancers. Lee and coworkers (Lee, 1996; Lee et al, 1998) reported that $\mathrm{H}$-cadherin (identical to T-cadherin) acts as a tumor suppressor factor in breast cancer. Exogenous T-cadherin expression reduced in vitro invasiveness of breast cancer cells. The $T$-cadherin gene was inactivated in a considerable number of primary lung cancers by hypermethylation of the promoter region (Sato et al, 1998a). Toyooka et al (2001) also demonstrated that frequent aberrant methylation of $\mathrm{CDH} 13$ (identical to T-cadherin) in breast and lung cancers was accompanied by loss of gene expression. More recently, aberrant methylation of $C D H 13$ (T-cadherin) was found in $29 \%$ of bladder cancers, and methylation was associated with high tumor grade and nonpapillary growth pattern (Maruyama et al, 2001). These serial findings indicate that both gene deletion and aberrant methylation of promoter region are responsible for the down-regulation of T-cadherin gene expression and are related to malignant phenotype change in various cancers.

In the present study, we examined whether loss of heterozygosity $(\mathrm{LOH})$ and aberrant methylation of the $T$-cadherin gene occurred in invasive cutaneous squamous cell carcinoma. We used a polymorphic marker located in intron 1 of the T-cadherin gene for the $\mathrm{LOH}$ assay (Sato et al, 1998b). The results are summarized in Table 1B. LOH of the T-cadherin gene locus was observed in $8(28.5 \%)$ of 28 informative cases of squamous cell carcinoma (Fig. 4). LOH was also detected in two of five actinic keratosis components from which invasive squamous cell carcinoma arose. In contrast, $\mathrm{LOH}$ was not found in any of the eight cases of Bowen's disease or in the seven cases of actinic keratosis tested in which T-cadherin was expressed and a squamous cell carcinoma component was not found. The entire genomic DNA of the promoter region and 14 exons including exon-intron

Table 1. T-Cadherin Expression (A) and Relation Between $\mathrm{LOH}$ in Intron 1 and Methylation Status (B) in Actinic Keratosis, Bowen's Disease, and Invasive Cutaneous Squamous Cell Carcinoma

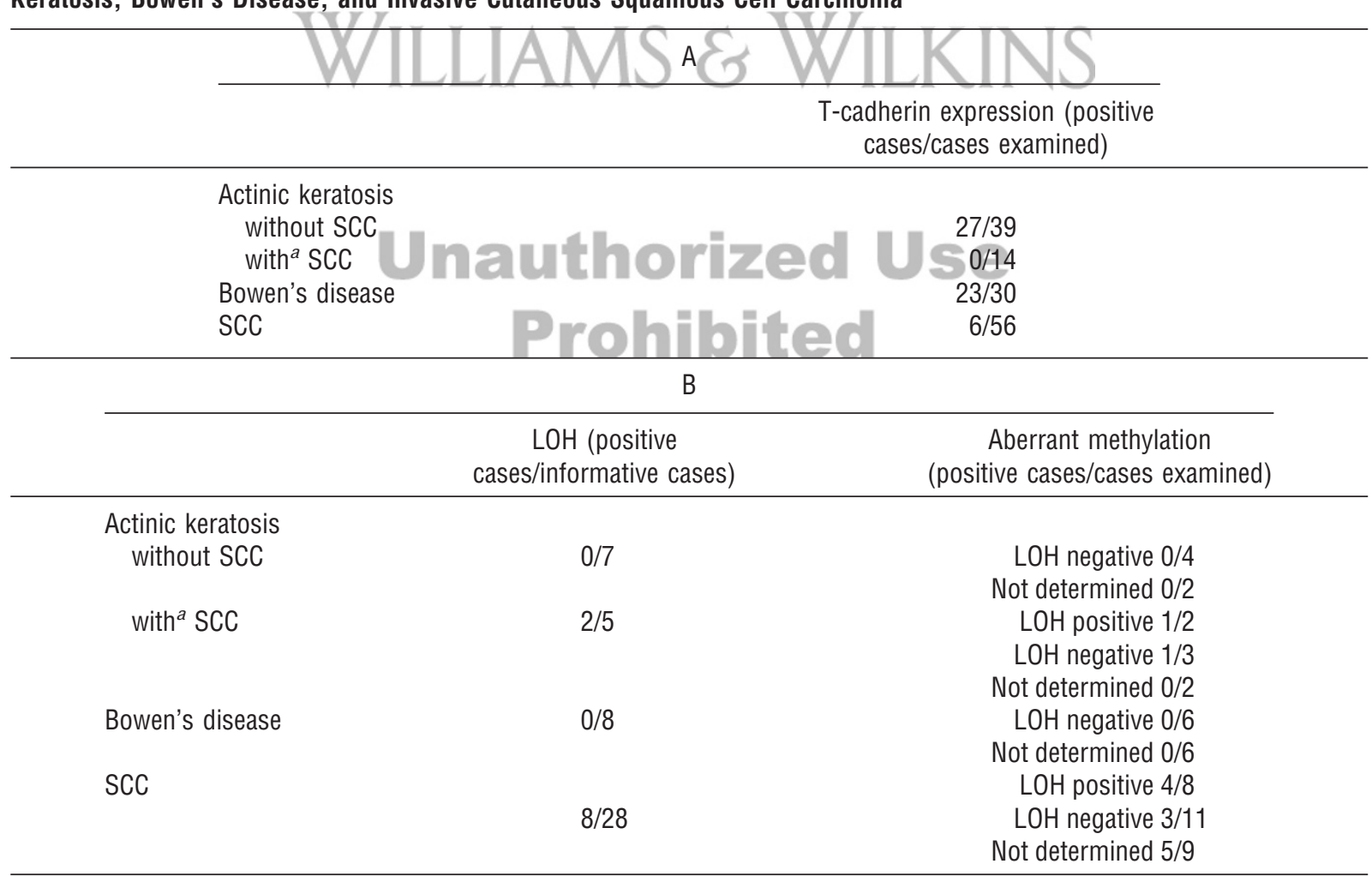

LOH, loss of heterozygosity; SCC, squamous cell carcinoma.

${ }^{a}$ Actinic keratosis components, from which invasive SCC arose, was investigated. 


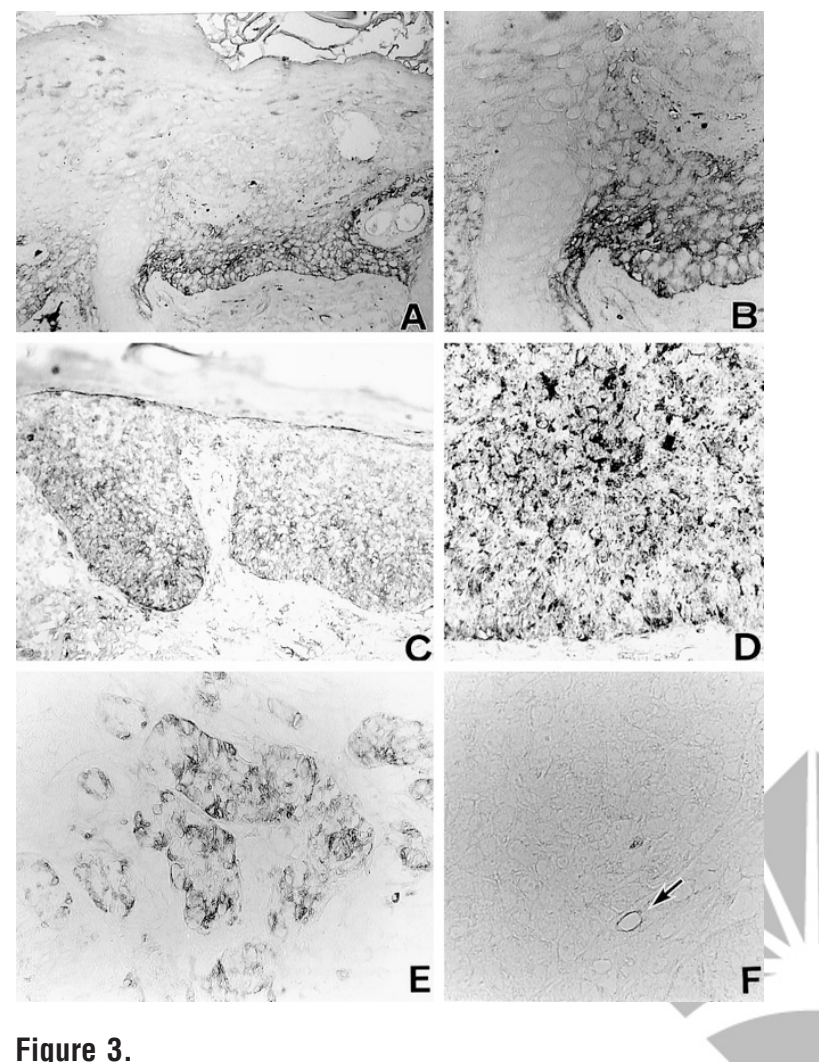

Figure 3.

Immunohistochemical staining of skin specimens of actinic keratosis, Bowen's disease, and cutaneous squamous cell carcinoma. Representative T-cadherin expression in actinic keratosis ( $A$ and $B$ ), Bowen's disease ( $C$ and $D)$, and invasive squamous cell carcinoma $(E$ and $F$ ) was demonstrated. Atypical keratinocytes in 27 of 53 actinic keratosis specimens were stained with anti-T-cadherin antibody. Most tumor cells in 23 of 30 Bowen's disease were also stained with anti-T-cadherin antibody. In contrast, T-cadherin expression was observed in only 6 of 56 invasive squamous cell carcinoma specimens (E). Fifty of 56 invasive squamous cell carcinoma specimens did not express T-cadherin $(F)$. Note the T-cadherin expression in vascular endothelial cells (arrow). Original magnifications, $\times 200(A$ and $C) ; \times 400(B, D, E$, and $F)$.

boundaries of the T-cadherin gene were sequenced in 7 cases of invasive squamous cell carcinoma in which we did not detect T-cadherin expression and in which we found $\mathrm{LOH}$ in intron 1 of the $T$-cadherin gene. No mutation was found in seven invasive squamous cell carcinoma specimens. A single-base substitution of $G$ to $A$ (tcg to tca, codon 69) at exon 3 or A to $G$ (caa to cag, codon 288) at exon 7 was found in three of seven specimens. However, neither substitution resulted in amino acid change. We found not only the identical substitution in genomic DNA obtained from nontumorous regions of skin from identical patients but also the expressed sequence tag cDNAs that contain the same substitution in the public database. We therefore concluded that both one-base substitutions are polymorphisms rather than point mutations.

Next, we examined whether aberrant hypermethylation of the promoter region of the $T$-cadherin gene occurred in squamous cell carcinomas. Genomic DNAs were isolated from paraffin-embedded tissue sections, modified with sodium bisulfite, and used as a template for methylation-specific PCR (MSP) as described by Herman et al (1996). In this assay, nonmethylated cytosines $(\mathrm{C})$ were converted to uracils

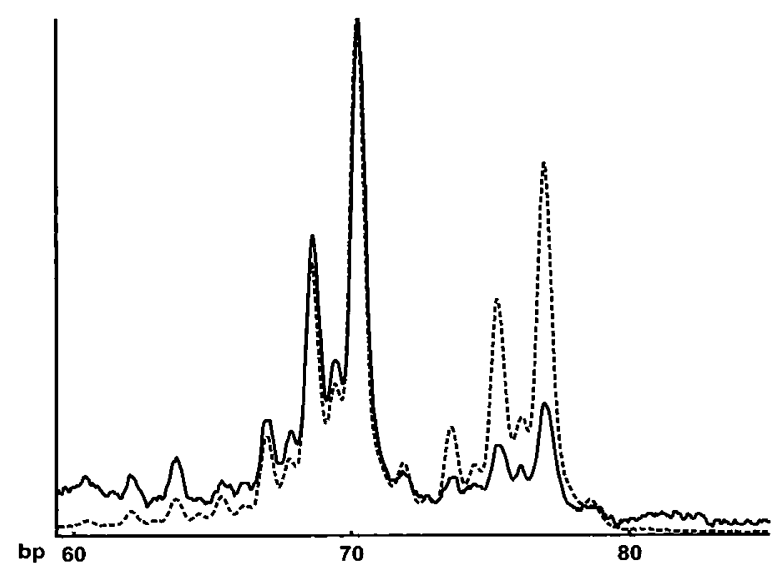

Figure 4.

Representative example of a clonal deletion of $T$-cadherin gene in a Tcadherin-negative squamous cell carcinoma. Loss of heterozygosity (LOH) in an invasive squamous cell carcinoma specimen that lacked T-cadherin expression is demonstrated. The genotype from tumor-rich areas is shown as a solid line. For comparison, the genotype derived from tumor-free areas of an identical patient are superimposed (dotted line). Immunohistochemical staining of this specimen is demonstrated in Figure 3F.

(U) and ultimately detected as thymidines (T) after PCR with unmethylation-specific primers, whereas methylated cytosines remained as cytosines and were subsequently amplified with methylation-specific primers. As demonstrated in Figure 5, PCR products with methylation-specific primers using bisulfite-modified genomic DNAs were detected in 4 of 8 and 3 of 11 $\mathrm{LOH}-$ positive and LOH-negative squamous cell carcinoma specimens, respectively. The aberrant methylation was also detected in five of nine squamous cell carcinoma specimens in which $\mathrm{LOH}$ was not determined. The relation between $\mathrm{LOH}$ and aberrant methylation status is summarized in Table 1B.

MSP assay also demonstrated hypermethylation in a cultured epidermoid carcinoma cell line of the vulva, A431 (Giard et al, 1973), but not in DJM-1. We further examined hypermethylation status in cultured squamous cell carcinomas by treating the cells with the demethylating agent 5'-aza-2-deoxycytidine (AzaCdR) (Bender et al, 1998). Incubating the cells with 2 $\mu \mathrm{g} / \mathrm{ml}$ Aza-CdR for 6 days restored T-cadherin mRNA expression in A431 cells (Fig. 6). These findings indi-

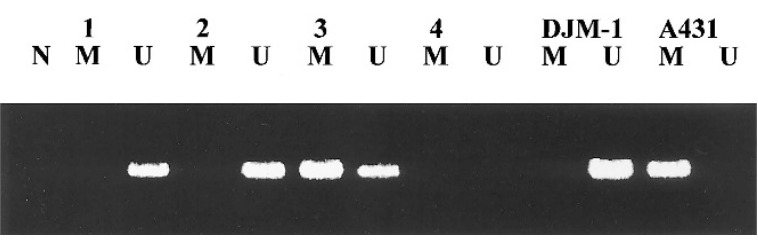

Figure 5.

Representative examples of MSP analyses. Primer sets used for amplification are designated as unmethylated $(U)$ or methylated $(M)$. DNA from tumor-rich areas of four squamous cell carcinomas specimens, numbered 1 to 4 , was modified by bisulfite and used as a template. Note the bands of the methylated form of $T$-cadherin gene in case 3 . In case 4 , neither methylation-specific nor unmethylation-specific bands were detected and counted as an uninformative case. A methylation-specific band was also found in A431 cells but not in DJM-1 cells. Negative control without genomic DNA is shown in lane $N$. 


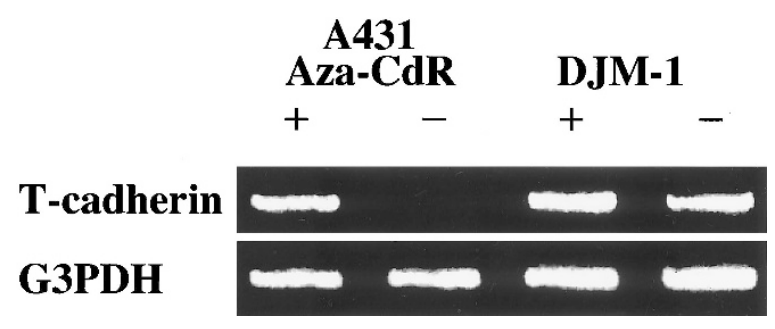

Figure 6.

Restoration of T-cadherin expression after $5^{\prime}$-aza-2-deoxycytidine (Aza-CdR) treatment. Human epidermoid cell carcinoma A431 and squamous cell carcinoma of skin DJM-1 cultured cells were incubated with Aza-CdR. Restoration of T-cadherin expression was observed in A431 cells by RT-PCR. Note the even intensity of internal control bands of G3PDH.

cated that down-regulation of T-cadherin in invasive cutaneous squamous cell carcinoma was caused by the combination of $\mathrm{LOH}$ and hypermethylation, as reported for breast and lung cancer. However, it should also be noted that there were T-cadherin negative squamous cell carcinoma specimens in which neither allelic loss nor aberrant hypermethylation was detected. It is possible that other factors participate in T-cadherin down-regulation in invasive squamous cell carcinoma.

In conclusion, the present study demonstrated that expression of a novel cadherin molecule, T-cadherin, was down-regulated in cutaneous invasive squamous cell carcinoma. The combination of allelic loss and hypermethylation may be an important mechanism of inactivation of the T-cadherin gene in invasive squamous cell carcinoma. We also speculate that reduction of T-cadherin expression may precede dermal invasion of squamous cell carcinoma.

\section{Materials and Methods}

\section{Antibodies and Immunohistochemical Staining}

Details of the procedures used to prepare rabbitspecific antibody to human T-cadherin and immunohistochemical staining were described previously (Takeuchi et al, 1999). Briefly, rabbits were immunized with human T-cadherin peptide, QRQPFPRDVGKVVDSDRPERSKFRLTGKGVD, according to the method of Lee (1996). Antibody was purified by affinity chromatography using the same peptide. Control normal rabbit IgG was also prepared in our laboratory. In the present study, we used autoclave pretreatment for antigen retrieval. Deparaffinized sections were placed first in plastic Coplin jars filled with citrate buffer $(\mathrm{pH}$ 6.0) and incubated for $15 \mathrm{~min}$ at $120^{\circ} \mathrm{C}$ in an autoclave. Then, the tissues were immunostained with affinity-purified anti-human T-cadherin peptide or control antibody using a streptavidin biotin complex peroxidase kit (DAKO LSAB Kit; Dakopatts, Kyoto, Japan). In several experiments, anti-T-cadherin antibody was preadsorbed with human T-cadherin peptide, which was used for immunization.

\section{Carcinoma Cell Lines and Human Skin Tissue Equivalent}

Two squamous cell carcinoma cell lines, DJM-1 and A431, derived from human skin and vulva, respectively, were obtained from RIKEN Cell Bank (Tsukuba, Japan) and maintained in our laboratory with DMEM supplemented with $10 \%$ fetal bovine serum. TESTSKIN Living Skin Equivalent was obtained from TOYOBO Biochemicals (Osaka, Japan). TESTSKIN was stored in an incubator at $36^{\circ} \mathrm{C}$, and the special packaging in which it was shipped was opened immediately before use.

\section{Western Immunoblotting}

Western immunoblotting was performed according to the modified method of Towbin et al (1979), as previously reported (Takeuchi et al, 2000). Briefly, extracted protein mixtures of cultured TESTSKIN were prepared with a lysis buffer $(20 \mathrm{mM}$ Tris-HCl, $0.01 \%$ SDS, $0.5 \%$ Nonidet P-40, 1 mM EDTA, 1 mM phenylmethyl sulfonyl fluoride, $5 \mu \mathrm{g} / \mathrm{ml}$ aprotinin). Extracted protein mixture from human heart was purchased (Clontech, Palo Alto, California). Equal amounts of proteins were subjected to electrophoresis on SDS-PAGE gels and electroblotted to a nitrocellulose membrane. After blocking with fetal bovine serum, membranes were incubated with affinity-purified rabbit antibody to human T-cadherin or normal rabbit IgG. Membranes were then incubated with a second antibody labeled with horseradish peroxidase and developed with diaminobenzidine.

\section{LOH Analysis}

The procedūre used was described in detail previously (Takeuchi et al, 2001b). Briefly, DNA was extracted from both tumor-rich and tumor-free areas of tissue sections from patients with cutaneous invasive squamous cell carcinoma using the Pinpoint Slide DNA Isolation System (Zymo Research, Orange, California) in accordance with the manufacturer's protocol. We selected tumor-rich specimens in which the proportion of tumor component was estimated to be at least $80 \%$. Extracted DNA was examined for genetic alternations using a GT dinucleotide repeat polymorphic marker in intron 1 of the T-cadherin gene. According to the method of Sato et al (1998b), PCR amplification was performed with the primers $5^{\prime}$-TCCCACTCTGGCGTGCGT-3' (sense) and 5'-TCCTCCCGTTAACGAACGTA-3' (antisense). Sense primer only was fluorescently labeled at the $5^{\prime}$ end. Template genomic DNAs (100 ng) were amplified by PCR using a thermal cycler (Takara Co. Ltd., Ohtsu, Japan) in a volume of $50 \mu$ containing $1.5 \mathrm{mmol} / \mathrm{L}$ of $\mathrm{MgCl}_{2}, 200 \mathrm{mmol} / \mathrm{L}$ of each dNTP, 50 pmol of each primer, and $2.5 \mathrm{U}$ of Taq polymerase (Perkin Elmer, Norwalk, Connecticut). PCR consisted of (1) initial denaturation at $95^{\circ} \mathrm{C}$ for 2 minutes; and (2) 35 cycles of $95^{\circ} \mathrm{C}$ for 30 seconds, $55^{\circ} \mathrm{C}$ for 30 seconds, and $72^{\circ} \mathrm{C}$ for 30 seconds. PCR products were run and analyzed using an automated fluorescent DNA sequencer (Amersham Pharmacia Biotech, Upsala, Sweden) on a $6 \%$ polyacrylamide 
denaturing gel. Allele ratios were calculated as principally described by Cawkwell et al (1993). Because incomplete losses are commonly observed in tumor and may reflect either normal cell contamination or tumor heterogeneity, a ratio of 0.50 was considered indicative of $\mathrm{LOH}$.

\section{DNA Sequencing}

Genomic DNA was extracted from paraffin tissue sections using the Pinpoint DNA Isolation System. The promoter region and 14 exons including exon and intron boundaries were amplified with primer sets as described by Sato et al (1998a). PCR conditions were also as described by Sato et al (1998a). Amplified PCR products were sequenced using an ABI PRISM DNA sequencer (PE Applied Biosystems, Foster City, California) as previously described (Takeuchi et al, 2001b).

\section{Analysis of Methylation Status}

For the MSP assay, genomic DNA was obtained from paraffin tissue sections that contained both cancerous and noncancerous areas with the Pinpoint Slide DNA Isolation System. Genomic DNA was modified with sodium bisulfite according to the methods of Herman et al (1996) and Fan et al (2002). Primers sets reported by Sato et al (1998a) were used for MSP. Briefly, genomic DNA was suspended in $45 \mu \mathrm{l}$ of $10 \mathrm{mM}$ TRIS-HCl (pH 7.4)/0.1 mM EDTA with $5 \mu \mathrm{I} 2 \mathrm{~N} \mathrm{NaOH}$ at $37^{\circ} \mathrm{C}$ for 15 minutes. A $1-\mu \mathrm{g}$ portion of salmon sperm DNA (Sigma-Aldrich Chemie GmbH, Steinhern, Germany) was added as a carrier before modification. Denatured DNA was modified by treatment with sodium bisulfite (Sigma-Aldrich Chemie $\mathrm{GmbH}$ ), which converts unmethylated cytosines to uracil but does not change methylated cytosines at $50^{\circ} \mathrm{C}$ for 48 hours. The modified DNA was purified using a Wizard DNA purification kit (Promega, Madison, Wisconsin), treated with $\mathrm{NaOH}$ to desulfonate and precipitated with ethanol. The forward and reverse primers for the methylated sequence were 5'-TCGGGGGGTTCGTIITTCGC-3' and 5'-GACGTTTTCATTCATACACGCG-3', respectively, whereas those for the unmethylated sequence were $5^{\prime}-$ TTGTGGGGTTGTIITTGT-3' and 5'-AACTTITCATTCATACACACA-3', respectively. PCR was performed as follows: $94^{\circ} \mathrm{C}$ for 2 minutes for initial denaturation followed by 35 cycles of $94^{\circ} \mathrm{C}$ for 30 seconds, $55^{\circ} \mathrm{C}$ for 30 seconds, and $72^{\circ} \mathrm{C}$ for 30 seconds. Final elongation at $72^{\circ} \mathrm{C}$ for 5 minutes was also performed. PCR products were subjected to electrophoresis in $2 \%$ agarose gels.

\section{Aza-CdR Treatment}

A431 and DJM-1 cells were incubated in culture medium with and without Aza-CdR at a concentration of $2 \mu \mathrm{g} / \mathrm{ml}$ for 6 days. Cells were harvested at the end of the sixth day for extraction of total RNA by using RNA-zol B (Biotex Laboratory, Houston, Texas). We used total RNA as substrate for CDNA synthesis with an RT-PCR kit (Life Technologies, Gaithersburg, Maryland) as previously described (Takeuchi et al, 2000).
The cDNA synthesis and subsequent PCR were performed according to the manufacturer's instructions. The primer sets used in this study were sense $5^{\prime}$ GCCACGATCATGATCGATGAC-3' and antisense 5'GTCTTCATTTTCCACTTTGA-3' for T-cadherin and sense 5'-TCCACCACCCTGTTGCTGTA-3' and antisense 5'-ACCACAGTCCATGCCATCAC-3' for G3PDH.

\section{References}

Bender CM, Pao MM, and Jones PA (1998). Inhibition of DNA methylation by 5-aza-2'-deoxycytidine suppresses the growth of human tumor cell lines. Cancer Res 58:95-101.

Cawkwell L, Bell SM, Lewis FA, Dixon MF, Taylor GR, and Quirke P (1993). Rapid detection of allele loss in colorectal tumours using microsatellites and fluorescent DNA technology. Br J Cancer 67:1262-1267.

Cerri A, Favre A, Giunta M, Corte G, Grossi CE, and Berti E (1994). Immunohistochemical localization of a novel $\beta_{1}$ integrin in normal and pathologic squamous epithelia. $J$ Invest Dermatol 102:2247-2252.

Fan X, Inda MM, Tunon T, and Castresana JS (2002). Improvement of the methylation specific PCR technical conditions for the detection of $\mathrm{p} 16$ promoter hypermethylation in small amounts of tumor DNA. Oncol Rep 9:181-183.

Fredette BJ, Miller J, and Ranscht B (1996). Inhibition of motor axon growth by T-cadherin substrata. Development 122:3163-3171.

Giard DJ, Aaronson SA, Todaro GJ, Arnstein P, Kersey JH, Dosik H, and Parks WP (1973). In vitro cultivation of human tumors: Establishment of cell lines derived from a series of solid tumors. J Natl Cancer Inst 51:1417-1423.

Herman JG, Graff JR, Myohanen S, Nelkin BD, and Baylin SB (1996). Methylation-specific PCR: A novel PCR assay for methylation status of $\mathrm{CpG}$ islands. Proc Natl Acad Sci USA 93:9821-9826. L I

Kawakami M, Staub J, Cliby W, Hartmann L, Smith DI, and Shridhar V (1999). Involvement of H-cadherin (CDH13) on $16 q$ in the region of frequent deletion in ovarian cancer. Int $J$ Oncol 15:715-720.

Kitajima Y, Inoue S, and Yaoita HJ (1987). Effects of pemphigus antibody on the regeneration of cell-cell contact in keratinocyte cultures grown in low to normal $\mathrm{Ca}^{++}$concentration. Invest Dermatol 89:167-171.

Lee SW (1996). H-cadherin, a novel cadherin with growth inhibitory functions and diminished expression in human breast cancer. Nat Med 2:776-782.

Lee SW, Reimer CL, Campbell DB, Cheresh P, Duda RB, and Kocher O (1998). H-cadherin expression inhibits in vitro invasiveness and tumor formation in vivo. Carcinogenesis 19:1157-1159.

Llorens A, Rodrigo I, Lopez-Barcons L, Gonzalez-Garrigues M, Lozano E, Vinyals A, Quintanilla M, Cano A, and Fabra A (1998). Down-regulation of E-cadherin in mouse skin carcinoma cells enhances a migratory and invasive phenotype linked to matrix metalloproteinase-9 gelatinase expression. Lab Invest 78:1131-1142.

Maruyama R, Toyooka S, Toyooka KO, Harada K, Virmani AK, Zöchbauer-Müller S, Farinas AJ, Vakar-Lopez F, Minna JD, Sagalowsky A, Czerniak B, and Gazdar AF (2001). Aberrant promoter methylation profile of bladder cancer and 
its relationship to clinicopathological features. Cancer Res 61:8659-8663.

Nagafuchi A and Takeichi M (1988). Cell binding function of E-cadherin is regulated by the cytoplasmic domain. Eur Mol Biol Organ J7:3679-3684.

Pizarro A (2000). E-cadherin expression is frequently reduced in infiltrative basal cell carcinoma. J Dermatol 27:804-805.

Ranscht B and Dours-Zimmermann MT (1991). T-cadherin, a novel cadherin cell adhesion molecule in the nervous system, lacks the conserved cytoplasmic region. Neuron 7:391-402.

Sato M, Mori Y, Sakurada A, Fujimura S, and Horii A (1998a). The $\mathrm{H}$-cadherin $(\mathrm{CDH} 13)$ gene is inactivated in human lung cancer [Published erratum in Hum Genet 103:532,1998]. Hum Genet 103:96-101.

Sato M, Mori Y, Sakurada A, Fujimura S, and Horii A (1998b). A GT dinucleotide repeat polymorphism in intron 1 of the H-cadherin (CDH13) gene. J Hum Genet 43:285-286.

Takeuchi T, Misaki A, Chen BK, and Ohtsuki Y (1999). $\mathrm{H}$-cadherin expression in breast cancer. Histopathology 35 : 87-88.

Takeuchi T, Misaki A, Fujita J, Sonobe H, and Ohtsuki $Y$ (2001a). T-cadherin (CDH13, H-cadherin) expression downregulated surfactant protein $\mathrm{D}$ in bronchioloalveolar cells. Virchows Arch 438:370-375.
Takeuchi T, Misaki A, Liang S-B, Tachibana A, Hayashi N, Sonobe H, and Ohtsuki Y (2000). Expression of T-cadherin (CDH13, H-cadherin) in human brain and its characteristics as a negative growth regulator of EGF in neuroblastoma cells. J Neurochem 74:1489-1497.

Takeuchi T, Nicole S, Misaki A, Furihata M, Iwata J, Sonobe $\mathrm{H}$, and Ohtsuki $\mathrm{Y}$ (2001b). Expression of SMARCF1, a truncated form of SWI1, in neuroblastoma. Am J Pathol 158:663672.

Takeuchi $T$ and Ohtsuki $Y$ (2001c). Recent progress of T-cadherin (CDH13, H-cadherin) research. Histol Histopathol 16:1287-1293.

Toyooka KO, Toyooka S, Virmani AK, Sathyanarayana UG, Euhus DM, Gilcrease M, Minna JD, and Gazdar AF (2001). Loss of expression and aberrant methylation of the $\mathrm{CDH} 13$ ( $\mathrm{H}$-cadherin) gene in breast and lung carcinomas. Cancer Res 61:4556-4560.

Towbin H, Staehelin T, and Gordon J (1979). Electrophoretic transfer of proteins from polyacrylamide gels to nitrocellulose sheets: Procedure and some applications. Proc Natl Acad Sci USA 76:4350-4354.

Zhou S, Matsuyoshi N, Liang SB, Takeuchi T, Ohtsuki Y, and Miyachi $Y$ (2002). Expression of T-cadherin in basal keratinocytes of skin. J Invest Dermatol 118:1080-1084.
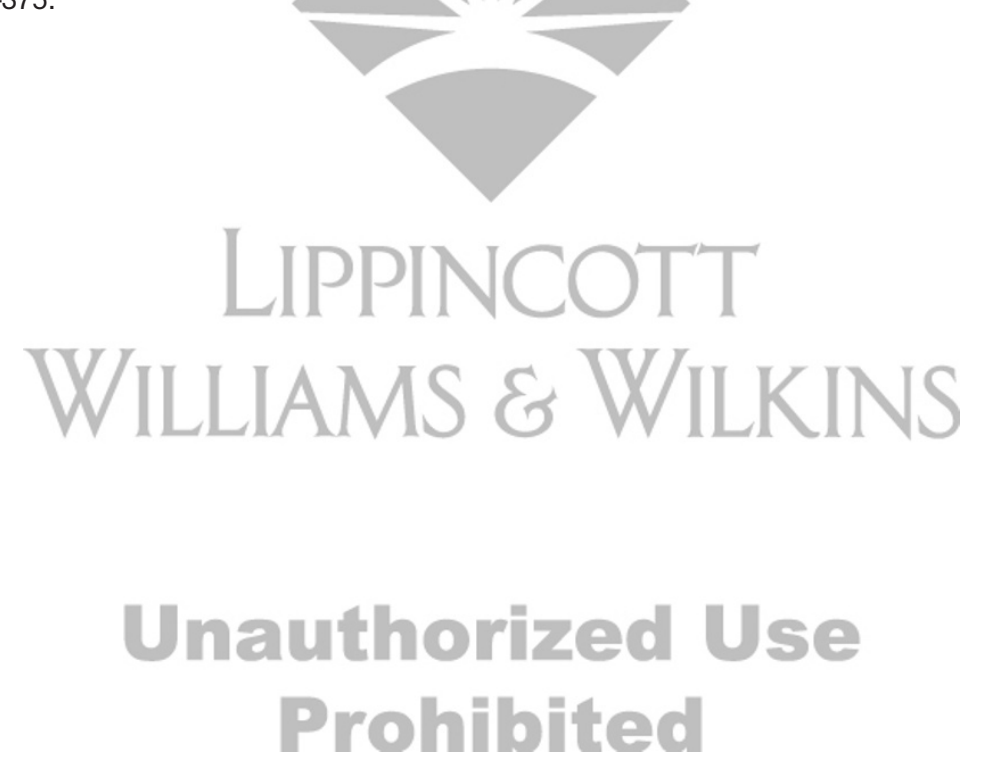